\title{
AudiTEE: Efficient, General-Purpose and Privacy-Preserving Audit for Distributed Ledgers
}

\author{
Zhufeng Ye, Zhenghao Wu, Xianglan Tian \\ College of Cyber Security, Jinan University, Guangzhou, China \\ Email: allenye1996@foxmail.com
}

How to cite this paper: Ye, Z.F., Wu, Z.H. and Tian, X.L. (2021) AudiTEE: Efficient, General-Purpose and Privacy-Preserving Audit for Distributed Ledgers. Journal of Computer and Communications, 9, 103-120. https://doi.org/10.4236/jcc.2021.98007

Received: July 30, 2021

Accepted: August 28, 2021

Published: August 31, 2021

Copyright ( 2021 by author(s) and ScientificResearch Publishing Inc. This work is licensed under the CreativeCommons Attribution International

License (CC BY 4.0).

http://creativecommons.org/licenses/by/4.0/

\begin{abstract}
Privacy-preservation and effective auditing are two desirable but challenging requirements on distributed ledgers. To meet the requirements, this paper presents an auditing scheme, called as AudiTEE, which can audit a distributed ledger in a generic, efficient, and privacy-preserving manner. AudiTEE leverages Trusted Execution Environment (TEE) to generate confidential but auditable transactions and realize arbitrary, efficient and confidential audit on them. Unfortunately, TEE suffers from some inherent barriers and is itself not a complete solution for fast audit. To tackle these challenges, AudiTEE takes advantage of KAMT (K-anonymity Authentication Based on Merkle Tree) protocol for efficient management on account and user-defined anonymous transactions. Further, to achieve a complete and fast audit with unlinkability, TEE doesn't process through all but only a comparatively small part of transactions according to a special ktag attached on each transaction to ensure that a user cannot hide transactions from auditor even when auditor is blind with who is involved in each transaction on the ledger. Apart from the above, AudiTEE allows flexible control on user behaviors. We implement a concrete instance of AudiTEE under a bank setting and demonstrate the scalability with all its core functionalities.
\end{abstract}

\section{Keywords}

Distributed Ledgers, Trusted Execution Environments, Auditing, Privacy-Preservation, Authentication

\section{Introduction}

A distributed ledger [1] [2] is a general distributed database that exists across 
several locations or among multiple participants. As a result, it is decentralized to mitigate the need for a central authority or intermediary to process, validate or authenticate transactions. Its records are stored one after the other in a continuous ledger, but they can only be added when the participants reach some consensus.

Audit [3] [4] plays an indispensable role in deterring ledger malpractice from time to time ${ }^{1}$. In the financial sector, for example, auditors verify the correctness of the company statement with transaction records from banks [5]. In September 2020, the transaction regulators confirmed Luckin's falsification of financial and operational figures [6], removed Luckin from NASDAQ list. Audit is also required in many sectors such as government and charity organization [7] [8].

Due to their promising immutable data and public verifiability merits demonstrated in the auditing process, distributed ledgers have become a brand new sword in the practice of audit by pioneers such as the "Big Four" (Deloitte, PwC, EY and KPMG) [9]. In the schemes of the auditing process of distributed ledger, each ledger user is assigned with a unique ID, and can go to service providers, such as banks or brokers, to open its service account. Service providers will then perform operations (e.g., payment or exchanging) according to the request of a valid account. A regulator can impose account-based policy on user and an auditor can audit the service records to hold any misbehaved individual to account.

Although the auditing on distributed ledgers brings many advantages, it triggers concerns on several issues. 1) Privacy leakage: As auditing could be extremely challenging when an auditor is blind with which transactions belong to a user, the auditor is usually authorized to know all the transactions of the user, i.e., the auditor requires to know the linkability of transactions [10] [11] [12] [13]. This requirement significantly threatens user's privacy on transaction time and payment frequency. Even worse, auditors may need excessive power to decrypt confidential transactions sometimes [10] [11] [12]. 2) Limited functionalities: To preserve the privacy of transactions, zero-knowledge proof is usually adopted to realize confidential audit in the schemes [13] [14] [15] [16]. However, the schemes can merely conform to some specific tasks, such as payment limitation and correct taxation. 3) Inefficiency. An audit based on zero-knowledge proof usually costs much time for the cooperation between the auditor and the user. Besides, zkLedger [16] enables a single audit to involve all irrelevant transactions in a time period for preserving unlinkability. Thus, they are impractical for large-scale audit on massive transactions and users.

Trusted Execution Environment (TEE) can enhance the confidentiality and integrity of computations [17]-[22]. Particularly, hybridized TEE-blockchain systems demonstrate the potential of TEE in the distributed ledger. For example, Proof of Luck [23] leverages TEE's random number generator to mimic the

${ }^{1}$ Originally defined in finance as independent examination of financial information of any entity, the concept of audit has now expanded to encompass so many areas in the public and corporate sector. It generally refers to inspection or examination, of a process or quality system, to ensure compliance to requirements. 
original Proof of Work consensus in a low-latency, egalitarian and energy-saving way. Teechain [24] seeks to deal with the scalability of the notable Bitcoin blockchain [25] under the aegis of TEE. Notwithstanding, they don't focus on auditability. [26] provides a TEE-based method for efficient and privacy-protected policy compliance on distributed ledger. However, audit merely takes place in transaction verification. Regretfully, TEE-based authentication suffers from inherent obstacles: 1) TEE is generally constrained in trusted memory. For example, Intel SGX only has a trusted memory of $128 \mathrm{MB}$. Therefore, all accounts information cannot be put into the enclave for anonymous user authentication. 2) TEE in general lacks trusted time source. It cannot reliably determine the state of a user is fresh or stale. This can be misused by a malicious user to create transactions with invalid account. 3) Trusted memory usually has worse performance than untrusted memory [27] [28], thus slowing down the authentication process.

With regard to auditing, arbitrary audit function can be performed in plaintext speedily and the plaintext is only known to the enclave itself. However, TEE itself is not an efficient solution for unlinkable audit. When an auditor cannot determine who is involved in a transaction, a complete ${ }^{2}$ audit has to be performed with all transactions in a time period as a price to ensure no transaction belonging to its auditee is left out. The exclusion of unrelated transactions will happen in the enclave to render a correct audit result. This is, nonetheless, highly impractical towards large scale audit.

The present scheme AudiTEE aims to provide efficient and privacy-preserving auditing on distributed ledgers. Specifically, it first creates an intermediarybased ledger, e.g., bank-intermediated ledger in the financial community [12] [29] [30], which enables a set of intermediary parties, namely, service providers, to post transactions of their users on the distributed ledger. Secondly, it employs an effective authentication component based on Trusted Execution Environment (TEE) which makes sure service providers only serve authorized users in a privacy-preserving way. Thirdly, auditable but confidential transactions and efficient, general-purpose and privacy-preserving audit are performed inside TEE to ensure confidentiality.

AudiTEE adopts KAMT (K-anonymity Authentication Based on Merkle Tree) protocol as a key component to enable TEE in efficient and anonymous user authentication and high-speed privacy-concealed audit: 1) To engage in user authentication, TEE simply stores a single short hash string rather than all accounts information. This short string helps TEE to identify that an account is currently valid. Meanwhile, the anonymous authentication for a user only relies on the verification of Merkle path, which is much faster than signature-based method. 2) Our special ktag produced allows any complete audit to be performed without the loss of unlinkability while much more practical and efficient than processing through all transactions, like zkLedger [16], as only a comparatively small part of transactions on the ledger needs to get involved. This prop-

${ }^{2} \mathrm{~A}$ complete audit involves all transactions of a user in a specific time period. 
erty guarantees user privacy on when a transaction occurs or the frequency of transaction while no relative transaction will be left out for an audit. Meanwhile, an audit can be performed immediately when permitted by a trusted authority and does not involve the cooperation with the auditee. Thus, supported by KAMT protocol, AudiTEE realizes complicated, confidential but fast audit compared to others.

Our contributions can be summarized as follows:

- AudiTEE framework. We propose AudiTEE to support efficient, general-purpose and privacy-preserving audit on confidential distributed ledger.

- KAMT protocol. We introduce this special building block to provide TEEempowered solution for efficient identity management, user-defined anonymous authentication and enable fast and complete audit for AudiTEE.

- Implementation and Experiments. We have an instantiation of AudiTEE under bank setting and demonstrate its gratifying performance.

Outline of this work. The rest of this paper proceeds as follows. In Section 2, we propose AudiTEE scheme including the system model, security model and modules. In Section 3, we discuss the performance of AudiTEE. We instantiate AudiTEE under a banking scenario to examine its performance in Section 4 . The paper is concluded in Section 5.

\section{The Proposed Auditing Scheme}

AudiTEE provides efficient, general-purpose and privacy-preserving audit by integrating TEE-enabled hosts into distributed ledgers. It can achieve the following goals: 1) Privacy-preservation. The transactions of users are confidential and unlinkable. 2) Correctness: Only valid transactions can be produced. 3) Completeness: An audit cannot perform at the price of linkability although all relative transactions are checked. The notations are listed in Table 1.

Table 1. Notations.

\begin{tabular}{|c|c|}
\hline Variable & Definition \\
\hline $\begin{array}{l}\text { Merkle Verify (proof, } \\
\qquad, M R \text { ) }\end{array}$ & $\begin{array}{l}\text { Function to verify whether proof is the existential proof of leaf } v \text { with Merkle } \\
\text { root } M R \text {. }\end{array}$ \\
\hline $\begin{array}{c}\text { ktagVerify (proof, } \\
\text { ktag) }\end{array}$ & Function to verify whether ktag conforms to existential proof. \\
\hline (epk, esk) & Key pair for encryption use. \\
\hline (spk, ssk) & Key pair for signing use. \\
\hline$K$ & Symmetric key $K$. \\
\hline $\operatorname{sign}_{s s k}(\mathrm{~m})$ & To sign message $m$ with $s s k$. \\
\hline enc $c_{e p k}(m)$ & To encrypt message $m$ with $e p k$. \\
\hline$e n c_{K}(m)$ & To encrypt message $m$ with $K$. \\
\hline $\operatorname{rand}(\lambda)$ & To generate a randomness of length $\lambda$. \\
\hline$H(m)$ & $\begin{array}{l}\text { Collision-resistant hash function with } \\
\text { input } m \text {. }\end{array}$ \\
\hline
\end{tabular}




\subsection{System Model}

As shown in Figure 1, AudiTEE consists of four parties: regulator, service providers, users and auditors:

Regulator $R$ is the party to supervise the distributed ledger. It is in charge of 1 ) managing accounts for users and service providers; 2 ) enforcing policy on user behaviors; 3) judging the lawfulness of an auditing request from an auditor; and 4) helping the authorized auditor to get the result.

TEE-empowered Service Provider $P_{i}$ enrolls a user and receives his service requests through TEE, verifies the requests, and forms confidential and authenticated transactions inside a TEE.

User $U$ is at least enrolled by one $P_{i}$. He performs actions on the ledger through the TEEs of service providers as an intermediary and follows certain policy. His behaviors or transactions can be audited by an auditor under the permission of $R$.

Auditor $A$ is to inspect the transactions of users. Under the authorization of $R$, an auditing process takes place in the TEE of some $P_{i}$.

AudiTEE consists of 3 phases. In phase I, each user $U$ registers with some provider $P_{i}$ for an account under the confirmation of the regulator $R$. In phase II, user $U$ anonymously authenticates himself to the TEE of $P_{i}$ with KAMT protocol and then posts confidential transactions on the distributed ledger. In phase III, auditor $A$ sends the auditing request to $R$. As long as the auditing request is approved, auditor $A$ will carry on auditing process on $U$ s transactions inside the TEE of some $P_{i}$.

PHASE IIITEE)

2.Audit on users

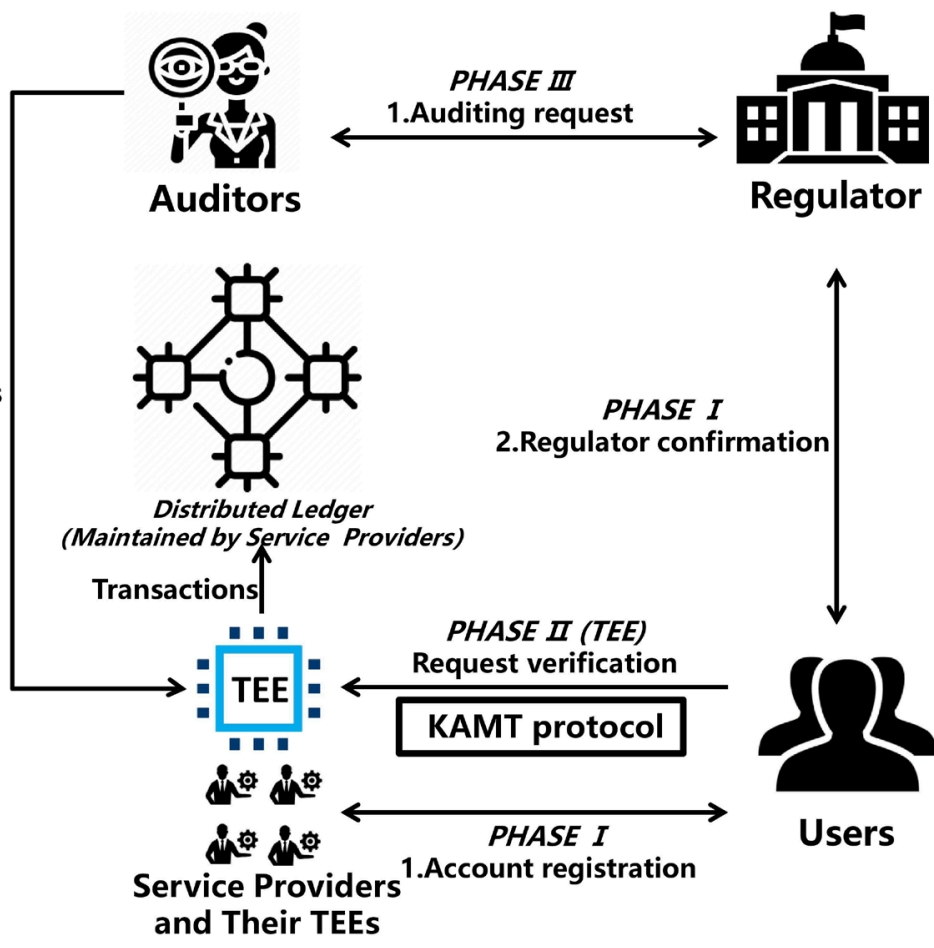

Figure 1. AudiTEE system model. Authentication and audit are performed inside the TEEs of service providers. 


\subsection{Security Model}

We now elaborate on assumptions of AudiTEE:

Regulator is semi-honest. It honestly follows the AudiTEE protocols and doesn't misuse the power of audit, but is curious about who is making a transaction.

Service Provider is considered to be dishonest if it: 1) helps malicious users to misbehave; 2) breaks the privacy of transactions.

User is assumed to be untrustworthy if he behaves in the following ways: 1) lies about his transactions during auditing; 2) makes a transaction against policy; 3) makes a transaction from an invalid account.

Auditor is semi-honest. She follows the AudiTEE protocols but is curious about user privacy during auditing.

AudiTEE doesn't protect against an adversary who performs network traffic analysis. For example, if $U$ sends a message to the TEE of $P_{i}$, it's reasonable to assume that a new transaction involves $U$.

\subsection{AudiTEE Protocol}

According to Figure 1, AudiTEE comprises of three modules, 1) initialization (phase I): initializes Merkle tree for user information; 2) authenticated transaction generation (phase II): generates the transaction and 3) privacy-preserving auditing (phase III): performs auditing process.

Initialization (Phase I). In the beginning, user $U$ registers with some provider $P_{i}$ under the confirmation of $R$ as shown in Figure 2. Specifically, user $U$ produces his asymmetric key pair $\left(e p k_{U}, e s k_{U}\right)$, and requests for registration to some provider $P_{i}$. Afterwards, $P_{i}$ signs $e p k_{U}$ and a timestamp $t s$ with its signing key $s s k_{P_{i}}$ as $\sigma_{P_{i}} . U$ sends a tuple $<s p k_{P,}, e p k_{U}, t s, \sigma_{P_{i}}>$ to $R$ for confirmation.

$R$ defines a user policy usrSpecif on user $U$, and forms user information info $=$ $H$ (epk $k_{U}$, usrSpecif, $r$ ), where $r$ is a nonce, and $H($.$) is a one-way hash function.$ info will be included into a publicly accessible Merkle tree maintained by the regulator $R$ as Figure 3. $R$ then returns to the user $U$ a tuple $<e p k_{U}$, usrSpecif, $r$, index $>$, where index is the position of leaf node for info. The Merkle tree will be re-produced by the regulator when there are some events including user join, user leave or info update.

Authenticated transaction generation (Phase II). To post a verifiable transaction on the distributed ledger, $U$ needs to authenticate himself through TEE in an anonymous and efficient manner using KAMT protocol.

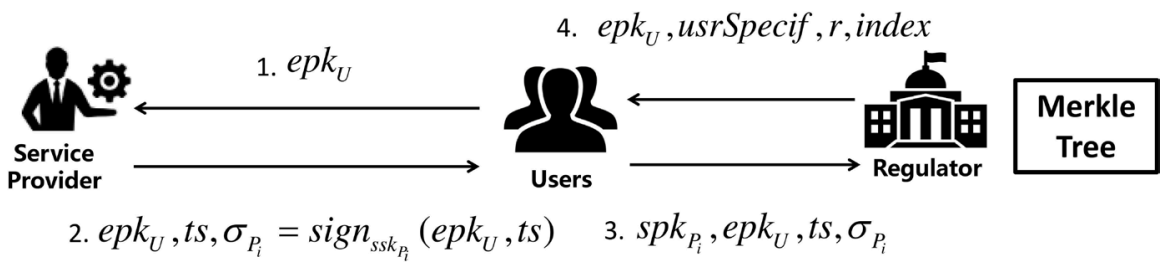

Figure 2. Registration process. 


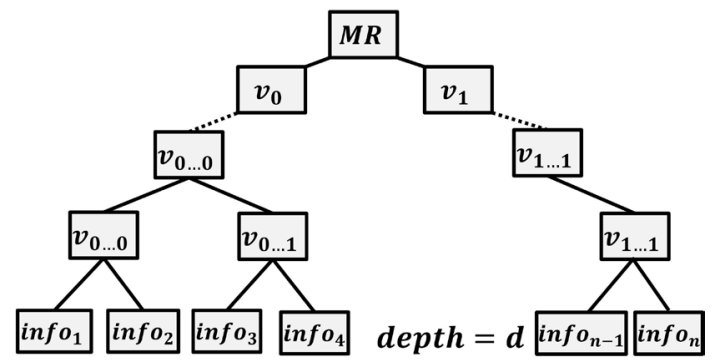

Figure 3. Identity management with Merkle tree. Any leaf node is a value info for a user or 0 , and any non-leaf node is $v_{y}=H\left(v_{y 0} / / v_{y 1}\right)$ for two children nodes $v_{y 0}$ and $v_{y 1}$.

Due to the Merkle tree update, $U$ needs to fetch the latest proof of his identity. Furthermore, in order to hide his identity in the retrieval, rather than asking for a specific proof, this can be done by blinding part of the original proof with self-defined $2^{m}$ leaves under a specific node amid the path from info to the root. Technically, we call the index of this specific node ktag, the subroot index of a subtree. For example, supposing $d=10$ (the tree is with $2^{9}$ leaves) and $m=6$, the retrieval of the Merkle proof of $V_{011101011}$ (a specific info) includes all $V_{011 x_{1} \ldots \times x,}$, where $x_{i} \in\{0,1\}$ and $\left|011 x_{4} \ldots x_{9}\right|=9$, and $\left(V_{010}, V_{00}, V_{1}\right)$. User can choose a desirable $2^{m}$ including enough non-empty info and easily recover its complete proof $\left(V_{011101011}, V_{011101010}, V_{01110100}, V_{0111011}, V_{011100}, V_{01111}, V_{0110}, V_{010}, V_{00}, V_{1}\right)$. The $k \operatorname{tag}$ for $U$ is 011 , indicating $U$ is some user under $V_{011}$. The design of KAMT protocol ensures ktag is bound with an existential proof and cannot be forged.

$P_{i}$ runs a TEE program which receives the Merkle tree root $M R$. Meanwhile, $U$ sends the tuple $<e p k_{U}$, usrSpecif, $r$, proof $>$ to TEE. By checking the recovered root value and retrieved root value $M R$, TEE determines whether the user is genuine or not.

Now we integrate KAMT protocol to the generation of authenticated transaction. We illustrate the whole process in Figure 4. Any genuine user $U$ authenticated sends request $t x R e q$ to $T E E_{P i}$, where $t x R e q$ including a pack of data that's required for service-specific logic, such as epk for $c_{p}$, ktag and other service-dependent data. For the validated request, $T E E_{P i}$ will forms an authenticated transaction $T X$ as described in Algorithm 1 in Figure 5. Table 2 is the authenticated transaction, where $c_{p}$ refers to secret information and $k t a g$ denotes an anonymous participant inside a group of accounts. For simplicity, $c_{p}$ is encrypted under one epk and there is only one $k t a g$ for a single anonymous user in a transaction. The transaction $T X$ is broadcast to the distributed ledger network for further validation such as double-spending checking.

Privacy-preserving audit (Phase III).For any user in the distributed ledger, AudiTEE can perform efficient, general-purpose and confidential auditing and will not leak any extra information.

Algorithm 2 in Figure 6 illustrates the auditing process. Auditor $A$ first sends a specific audit request on the user $U$ to the regulator $R$, which is presented as code AudProg. If the request is legal, $R$ informs the auditor $A$ all the leaves belonging to $U$. Then $A$ posits the transactions' $c_{a}$, a set of $c_{a}$ with ktag involving 


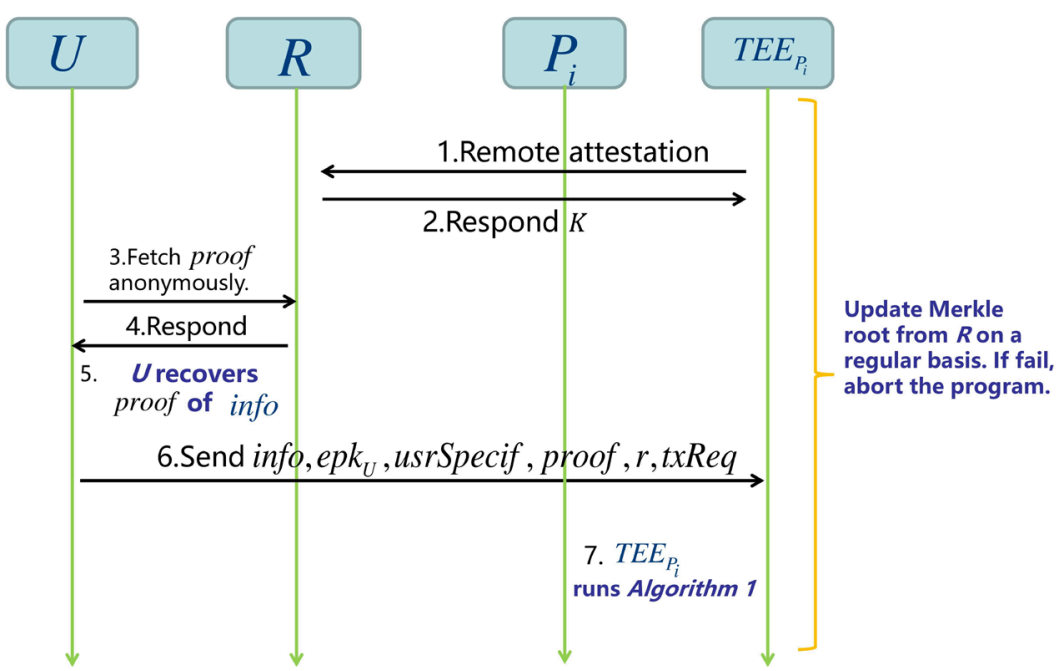

Figure 4. Generation of authenticated transaction.

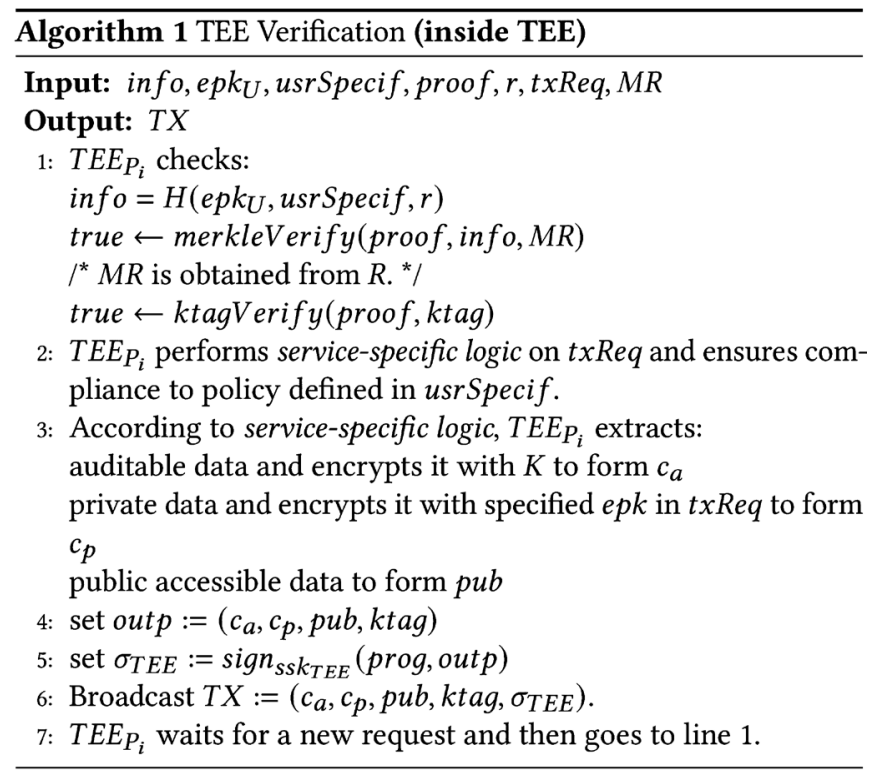

Figure 5. Algorithm 1 for TEE verification.

Table 2. Transaction in AudiTEE.

\begin{tabular}{cl}
\hline & \multicolumn{1}{c}{$T X=\left(c_{a}, c_{p}\right.$, pub, ktag, $\left.\sigma_{T E E}\right)$} \\
\hline$c_{a}$ & Auditable contents encrypted under the symmetric key $K$ of $R$. \\
$c_{p}$ & Secret information for specific user. \\
$k u b$ & Publicly accessible information. \\
$k t a g$ & Tag to indicate anonymity set of accounts. \\
$\sigma_{T E E}$ & Proof of the correct formation of a transaction in TEE. \\
\hline
\end{tabular}

leaves of $U$ ( $U$ is under a subtree with subroot index of $k t a g$ ). All $c_{a}$ will be sent into the TEE of $P_{i}$ and decrypted with $K$. The plaintext of $c_{a}$ should generally include the participant for TEE to exclude any transaction irrelevant to $U$. TEE 


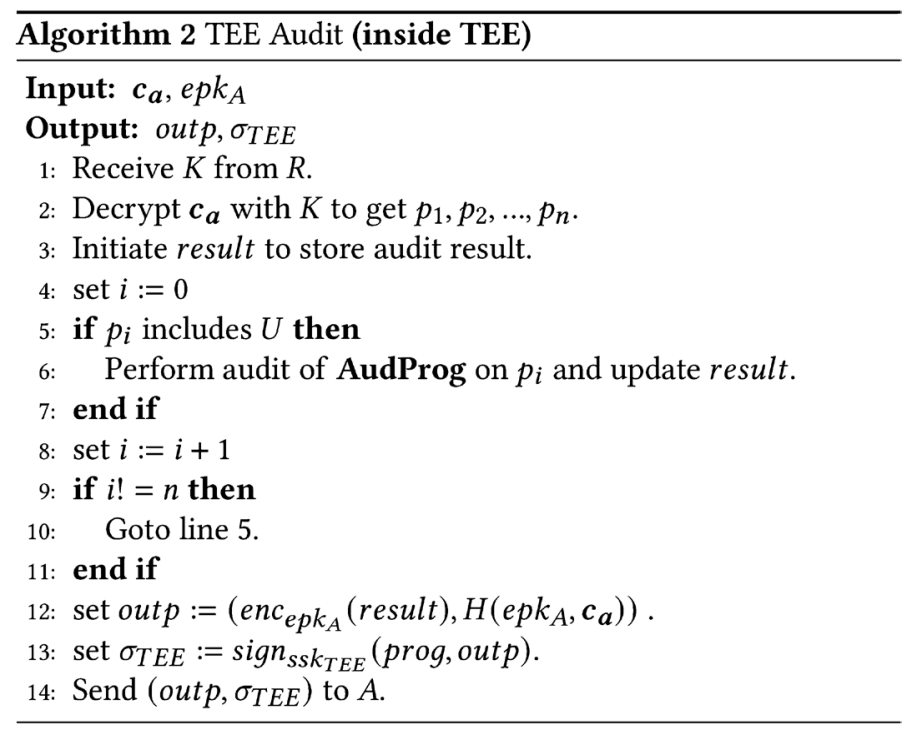

Figure 6. Algorithm 2 for TEE audit.

performs the operation defined in AudProg only on transactions of $U$. After the process in TEE, $A$ checks $\sigma_{T E E}$ to ensure the integrity of $c_{a}$ and the belonging of result by $H\left(e p k_{A}, c_{a}\right)$ and decrypts enc $c_{e p k A}$ (result) for the result.

\section{Performance Analysis of AudiTEE}

\subsection{Trade-off in Anonymity}

Although AudiTEE ensures user anonymity flexibly, side effect needs to be taken into consideration. Firstly, a transaction with higher anonymity will suffer from extra transaction delay due to network communication and recovery of Merkle proof. Meanwhile, stronger anonymity asks for more disk space to store the retrieved data before recovering the complete Merkle proof. What's more, extra burden may take place depending on a concrete scenario. For example, in our experimentation of a banking scenario in Section 4, the tracing of UTXO can be unwieldy when we choose a substantially safe $k t a g$ because such $k t a g$ is more likely to collide with other user's choice. All the above concerns may push normal user, e.g., mobile user, to choose a proper privacy-protecting level rather than the highest allowed and as a result, the process of audit can be faster. Thus, there is a trade-off between anonymity and audit efficiency.

\subsection{Quick Bootuping}

Algorithm 1, involving the encryption under regulator's key $K$, entails a bootup to recover $K$. Thus, a cooperation between $P_{i}$ and $R$ is demanded. In practice, thousands of TEEs may be run by a single $P_{i}$, requiring thousands of bootup cooperation. Also, unexpected breakdown of certain TEE will require a reboot. All these can pose a substantial burden to the whole system. We propose a knack to expedite the bootup process. At the onset, a TEE $T E E_{1}$ performs its bootup as usual. Ever since then, any new TEE, such as $T E E_{2}$, to join in can simply authen- 
ticate itself to a random TEE which has already booted up, e.g., $T E E_{1}$. $T E E_{2}$ will prove that it's running in an enclave with homogeneous program with $T E E_{1}$ by remote attestation. If successful, $T E E_{1}$ will directly share $K$ with $T E E_{2}$. This protocol doesn't require further participation of $R$ and can be quite effective when a cluster of TEEs are managed by a single party, like a bank.

\subsection{Optimization of TEE}

An optimization makes use of an enclave-generated $s s k_{T}$ to sign any output and $e p k_{T}$ to encrypt input. So only one remote attestation is required for the generation of $\left(e p k_{T}, s p k_{T}\right)$ and the protocol can then be run repeatedly without attestation. Meanwhile, $U$ can directly send a message to TEE without key exchange for symmetric key.

\subsection{Secret-Sharing on Regulator's Secret}

To enhance robustness of AudiTEE, any secret-sharing scheme [31] [32] [33] can be adopted to distribute the regulator's secret $K$ into pieces for several regulators. Thus, a single party cannot decrypt user secret furtively and an audit needs to be granted by a majority of regulators to be effective.

\subsection{Privacy-Preserving}

Theorem 1. Assume that the remote attestation scheme of TEE and the digital signature used for TEE-generated (spk, ssk) are existentially unforgeable under chosen message attacks (EU-CMA), that TEE guarantees confidentiality and integrity and that the anonymity set for $R$ is properly chosen, user can enjoy self-defined anonymity.

Proof. As the transaction is formed inside TEE, the private contents of a transaction can be protected. Meanwhile, supposing a user will post a transaction immediately after fetching its existential proof, the user authentication protocol only tells that a transaction is made by one of the chosen leaf nodes under the subtree of $\mathrm{ktag}$.

\subsection{Correctness and Completeness}

Theorem 2. Assume that the remote attestation scheme of TEE and the digital signature used for TEE-generated (spk, ssk) are existentially unforgeable under chosen message attacks (EU-CMA), a user can form an authenticated transaction only when he owns a valid account and conforms to certain policy and auditor enjoys a complete audit on any user.

Proof. To break the correctness of AudiTEE, a malicious user has to generate $\left(e p k_{U}\right.$, usrSpecif, $r$ ) not existing in the Merkle tree maintained by $R$ but with an existential proof successfully leading to the root of the tree, $M R$.

The correct attachment of ktag helps auditor to target a set of transactions including but larger than those for its auditee. The design of Merkle tree ensures that the chosen ktag is bound with an existential proof. 
As the hash function $H(\cdot)$ is one-way, the forgery of proof and ktag is computationally infeasible.

\section{Experiment and Result}

\subsection{Experiment Design}

We evaluate the performance of AudiTEE under a bank application field, including parties of users, commercial banks, auditors and central bank. Several commercial banks, as the role of service providers will enroll users and enable their access to the payment system built up with distributed ledger under the confirmation of central bank. Bank account is in the form (epk $k_{U}, b_{a n k}, k t a g$ ) to receive payment and $b a n k_{U}$ refers to an identifier for the bank enrolled $U$. Central bank plays the role of regulator to take care of valid accounts in a Merkle tree and audit request needs to be granted by it to be effective. For simplicity, the order of transaction will be decided by the central bank with its signature. Table 3 shows the concrete parameters of transaction. As explained in Algorithm 3 in Figure 7, each bank will receive and process payment request from user (including an identity proof fetched from central bank and other service-dependent data) via its TEE, $T E E_{B i}$, and a half done transaction, $T X$, will be directly sent to the central bank from the enclave. The central bank will first verify the signature of TEE, $\sigma_{T}$, is authentic, the UTXO has not been spent and then include the hash of previous transaction, $h$, to complete the new transaction. Finally, it signs the new transaction to produce $\sigma_{C B}$. The settled transaction, $T X$, will be sent to each bank for another check on order correctness, UTXO freshness, signature of both TEE and central bank and stored locally.

\subsection{Implementation}

We implemented the above application of AudiTEE in C. All of our experiment is run on desktop with Intel (R) Core (TM) i5-7500 $3.40 \mathrm{GHz}$ CPU (SGX supported) and 32 GB RAM on Ubuntu 18.04. We detail each component in the following: 1) Cryptographic tools: Our prototype uses OpenSSL (Version 1.1.1)

Table 3. Parameter setting (banking).

\begin{tabular}{|c|c|}
\hline \multicolumn{2}{|r|}{$T X=\left(c_{a}, c_{p}, p u b, k t a g, \sigma_{T}, h, \sigma_{C B}\right)$} \\
\hline$c_{a}$ & $\begin{array}{l}\text { The secret of payer, payee and amount under the encryption of } K \text { for audit. } c_{a}=e n c_{K} \\
\left(e p k_{\text {payer }}, e p k_{\text {payee }}, V\right) \text {. }\end{array}$ \\
\hline$c_{p}$ & 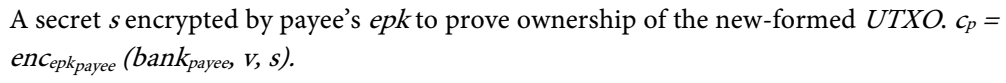 \\
\hline pub & The UTXO spent and a new-formed UTXO. UTXO $=H\left(e p k_{\text {payee }}\right.$, bank $\left._{\text {payee, }}, \mathrm{v}, \mathrm{ktag}, \mathrm{s}\right)$. \\
\hline ktag & A tag to indicate an anonymity account set of payee. \\
\hline$\sigma_{T}$ & Proof of the correct formation of a transaction in TEE (see Section 3.3). \\
\hline$h$ & The hash of prior transaction (added by central bank for order decision). \\
\hline$\sigma_{C B}$ & Proof of central bank to confirm the transaction. \\
\hline
\end{tabular}




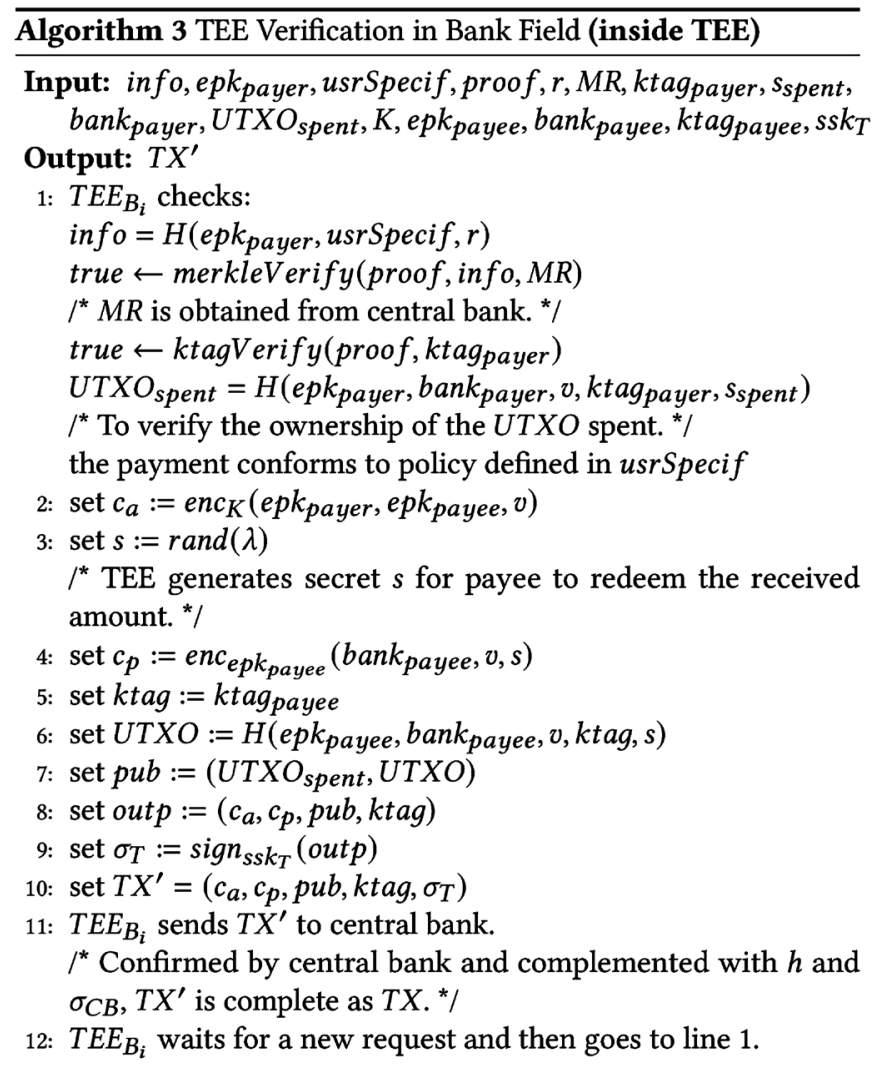

Figure 7. Algorithm 3 for TEE verification in bank field.

library for our cryptographic blocks. We choose SHA256 for our hash function, $A E S \_256 \_C B C$ with 256-bit key and 128-bit initialization vector for symmetric encryption. The PKCS \#1 v1.5 padding for asymmetric encryption and PKCS \#1 v2.0 for signing scheme are both with 1024-bit key. 2) Trusted Execution Environment: We use Intel SGX as our TEE component and the program is run with the support of Occlum library OS [34]. The server program is partitioned into trusted part, receiving, processing user request for a half done transaction (for central bank), and untrusted part, accepting and verifying final transaction from central bank and storing it locally. 3) Database and distributed ledger: Redis library is used as database for the storage of UTXOs for banks and central bank. It's also for the formation and maintenance of the Merkle tree for user management in the central bank side. Particularly, the subscriber-publisher model is adopted for the distribution of final transaction signed by central bank in a distributed ledger. We include central bank and commercial banks under a common channel but only the central bank is allowed to publish message (transaction) into the channel. Bank receives settled transaction from central bank and stores it locally. All honest banks will be consistent in their local ledgers. All the operation of Redis is controlled through Hiredis library.

Our evaluation responds to the following questions:

a) How expensive is it to manage identity in a Merkle tree (formation, update and retrieval)? (Experiment 1) 
b) What's the efficiency of transaction verification in AudiTEE and how does AudiTEE system scale with the number of banks? (Experiment 2)

c) What's the efficiency of different audit functions in online and offline mode? (Experiment 3)

d) What's the performance of UTXO tracing in online and offline mode? (Experiment 4)

Experiment 1 The first experiment concerns the maintenance of Merkle tree. Experiment 1 is divided into three parts: 1) Tree formation: During the construction of the tree, each user information epk, usrSpecif, $r$ (assuming 128 Bytes in total) will be hashed into info to become a leaf and then the tree will be fully calculated and stored locally. 2) Retrieval: In this part, some members of the tree will be fetched as that in Section 2.3. mix refers to number of the mixing accounts. 3) Update: In the update test, a specific info will be changed, entailing certain update of the tree. The first test was run once while the latter two run 10 , 000 times. We recorded the performance under different parameter setting.

According to our experiment outcome in Figure 8, the formation of the tree will grow exponentially with $k$ in time. We stress the formation of the tree, although most costly, is a one-time process for $R$. The fetching with anonymity set only grows slightly when $k$ increases. The exponentially growth of anonymity set leads to a similar growth in fetching time. The update of a leaf grows linearly when $k$ increases. We stress that all the above processes can be separated into several servers to boost velocity.

Experiment 2 The second experiment pertains to the performance of transaction verification and the overall throughput of AudiTEE. We first measure the verification component in both online and offline mode. Only one server with single thread is interacting with one client during online test. In offline test, we put all transaction requests locally and server simply fetches them from disk. Transaction verification confirms: 1) the request is from a valid account, 2) the ownership of UTXO spent; 3) the payment is correct and policy-conformed. More details have been included in Algorithm 3 in Figure 7. All the above tests were performed on mimic request for 1000 times online and 10,000 offline. Then
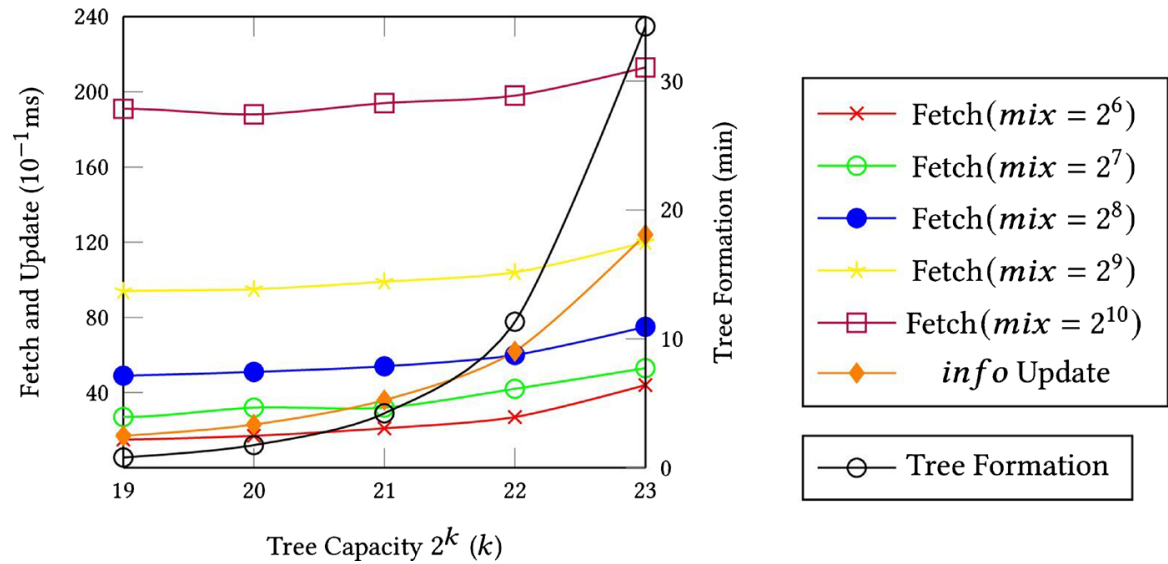

Figure 8. Merkle tree operation. 
we make things more integral, with central bank, banks and clients to have a more authentic measurement on its efficiency. Each server is run with four threads. To be emphasized, each socket between client and bank will be used only once to be more realistic. We adopt the optimization in Section 3.3 and all the data sent is encrypted under AES and RSA encryption except the broadcast of half done transaction from SGX and the settled one from central bank.

Table 4 shows the desirable speed of transaction verification. The comparison of online and offline mode points out the underuse of verification function. This may be resolved by an SGX shared by several server threads. The data required by verification and the transaction are both with desirable size. In Figure 9, the throughput result itself manifests its capability of high frequency application such as trading and exchange. As service provider is potential to be equipped with high-end server machine, the performance in practice can be more powerful than we've been shown.

Experiment 3 This experiment simulates various audit functions inside SGX. The auditing outcome contains the hash of $e p k_{A}$ and all input $c_{a}$ and the secret result under the encryption of $e p k_{A}$. Without loss of generality, we omit the process of remote attestation and recovery of $K$ inside SGX for their unnoticeable effect when transactions under audit are substantial. In offline mode, SGX will directly fetch desirable $c_{a}$ from the local. Online mode is required when the

Table 4. Transaction verification.

\begin{tabular}{|c|c|c|c|}
\hline Tree Capacity & $\begin{array}{l}\text { Data for Verification } \\
\text { (Plaintext/Ciphertext) }\end{array}$ & Online & Offline \\
\hline $2^{23}$ & $1.87 \mathrm{kB} / 2 \mathrm{kB}$ & $206 \mathrm{~ms} / \mathrm{tx}$ & $0.3 \mathrm{~ms} / \mathrm{tx}$ \\
\hline \multicolumn{2}{|c|}{ Halfdone Transaction Size } & \multicolumn{2}{|c|}{ Settled Size } \\
\hline \multicolumn{2}{|c|}{$0.39 \mathrm{kB}$} & \multicolumn{2}{|c|}{$0.55 \mathrm{kB}$} \\
\hline
\end{tabular}

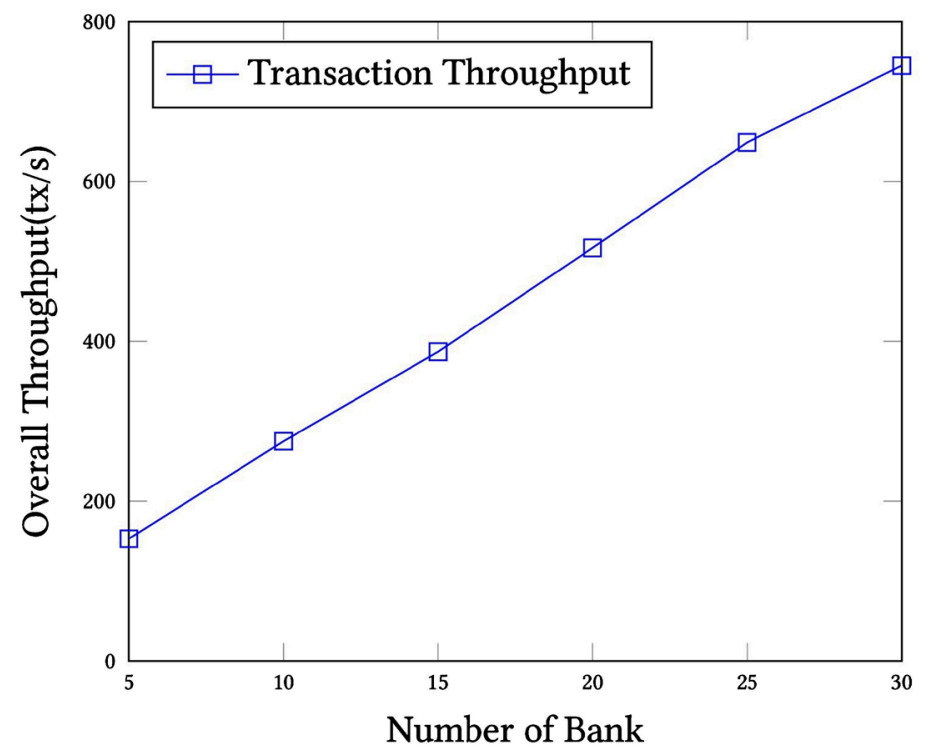

Figure 9. AudiTEE throughput. 
auditor asks for extra confidentiality. $c_{\boldsymbol{a}}$ will be sent under a symmetric key shared between bank's SGX and the auditor. As a result, bank can have no idea which transaction is currently being audited. We performed three types of audit: 1) summing (calculate balance of a user); 2) matching (how many transaction happened between two specified users); 3) exception detection (how many transaction of a user is beyond some value). Each audit type was performed online and offline for 1,000,000 times.

Table 5 shows a satisfactory speed for audit. Realistically, an audit only concerns relatively small part of $c_{a}$ on the ledger rather than all. What's more, several audit functions can actually be carried out in one audit and one audit can also be separately performed in several SGXs. Thus, there is still a considerable room for optimization.

Experiment 4 The final experiment is on the tracing of UTXO. In our implementation, UTXO is a hash commitment of $e p k_{U}$, bank identifier $b a n k_{U}$, value $v$, $k t a g$ and a secret randomness $s$. Bank $k_{U}, v, s$ are encrypted under the receiver's private key in $c_{p}$. Thus, to identify the ownership of a UTXO, the user first tries to decrypt $c_{p}$ to get $b_{a n k}, v, s$. Then it hashes, together with its $e p k_{U}$ and $k t a g$ to see if $U T X O=H\left(e p k_{U}, b_{a n k}, v, k t a g, s\right)$. Once again, the tracing may be carried out locally (offline mode), or can be passed from a bank to save disk space (online mode). Notice that this process is performed outside the SGX. Each mode was performed 1,000,000 times.

The outcome is shown in Table 6. A user only has to trace those transactions with its chosen $\mathrm{ktag}$. A ktag tells the possible recipients of a transaction. As ktag is self-defined as part of a user's account, (epk $k_{U}, b_{a n k_{U}}, \mathrm{ktag}$ ), to receive payment, when $\mathrm{ktag}$ indicates a larger subtree, thus with a higher anonymity, it's more likely that a transaction with user-chosen $\mathrm{ktag}$ may not actually belong to $U$, but other users under the same subtree. This means a lot of redundancy, or extra effort will take place when the possible transactions are to be transferred from a full node to and verified by user to confirm their real ownership. Thus, a user can properly choose a desirable ktag based on the cost of network communication and

Table 5. Transaction audit.

\begin{tabular}{cccc}
\hline & Data Size: 68 Bytes $/ \mathrm{tx}$ (offline) $/ 80$ Bytes $/ \mathrm{tx}$ (online) & \\
\hline Audit Type & Summing & Matching & Exception Detection \\
\hline Online & $0.017 \mathrm{~ms} / \mathrm{tx}$ & $0.016 \mathrm{~ms} / \mathrm{tx}$ & $0.017 \mathrm{~ms} / \mathrm{tx}$ \\
Offline & $0.002 \mathrm{~ms} / \mathrm{tx}$ & $0.002 \mathrm{~ms} / \mathrm{tx}$ & $0.002 \mathrm{~ms} / \mathrm{tx}$ \\
\hline
\end{tabular}

Table 6. UTXO tracing.

\begin{tabular}{cc}
\hline \multicolumn{2}{c}{ Data Size: 160 Bytes (offline)/180 Bytes (online) } \\
\hline UTXO Tracing & Time Cost \\
\hline Online & $0.095 \mathrm{~ms} / \mathrm{tx}$ \\
Offline & $0.088 \mathrm{~ms} / \mathrm{tx}$ \\
\hline
\end{tabular}


verification during the tracing of $U T X O$.

\section{Conclusion and Future Work}

AudiTEE proposes a practical framework for speedy, general-purpose and confidential audit on privacy-preserving distributed ledger. It can be implemented in various applications to enable user privacy with effective regulation. Powered by KAMT protocol, AudiTEE tackles several concerns for TEE in realising efficient account management in large scale. Meanwhile, the user-defined anonymous authentication for a user only relies on the verification of Merkle path, which is much faster than signature-based method. Specifically, a special ktag defined in KAMT protocol helps to realize fast audit on user while still preserving unlinkability and completeness. AudiTEE also allows various policies on user from regulator. Our evaluation shows that AudiTEE has satisfactory performance in all its core functionalities.

In the future, the current work can be extended in several aspects. Firstly, AudiTEE will be implemented in more applications for testing and improving. Secondly, extra attention should be focused on possible side-channel attacks on TEE and corresponding solutions. Finally, further research will be required to appease the need for a central trusted party, namely, regulator in AudiTEE system model to make the framework even more robust.

\section{Acknowledgements}

This work was in part supported by National Natural Science Foundation of China (Grant Nos. 61932011), Guangdong Basic and Applied Basic Research Foundation (Grant No. 2019B1515120010), Guangdong Key R\&D Plan2020 (No. 2020B0101090002), Key-Area Research and Development Program of Guangdong Province (No. 2020B0101090004) and Special Funds for the Cultivation of Guangdong College Students' Scientific and Technological Innovation ("Climbing Program" Special Funds.) (No. PDJH2021A0050).

\section{Conflicts of Interest}

The authors declare no conflicts of interest regarding the publication of this paper.

\section{References}

[1] Maull, R., Godsiff, P., Mulligan, C., Brown, A. and Kewell, B. (2017) Distributed Ledger Technology: Applications and Implications. Strategic Change, 26, 481-489. https://doi.org/10.1002/jsc.2148

[2] Rauchs, M., Glidden, A., Gordon, B., Pieters, G.C., Recanatini, M., Rostand, F., Vagneur, K. and Zhang, B.Z. (2018) Distributed Ledger Technology Systems: A Conceptual Framework. https://doi.org/10.2139/ssrn.3230013

[3] Wikipedia Audits. https://en.wikipedia.org/wiki/Audit

[4] American Society for Quality. What Is Auditing? https://asq.org/quality-resources/auditing 
[5] Investor.gov. The Laws That Govern the Securities Industry. https://www.investor.gov/introduction-investing/investing-basics/role-sec/laws-gov ern-securities-industry

[6] REUTERS (2020) China Fines Luckin Coffee and Linked Firms a Total of \$9 Million.

https://www.reuters.com/article/us-luckin-coffee-investigation-fine-idUSKCN26D0 $\underline{7 \mathrm{G}}$

[7] IRS Charity and Nonprofit Audits. https://www.irs.gov/charities-non-profits/exempt-organizations-audit-process

[8] AICPA What Is a Governmental Audit? https://www.aicpa.org/interestareas/governmentalauditquality/information-on-gov ernmental-audits.html

[9] Schmitz, J. and Leoni, G. (2019) Accounting and Auditing at the Time of Blockchain Technology: A Research Agenda. Australian Accounting Review, 29, 331-342. https://doi.org/10.1111/auar.12286

[10] Mitani, T. and Otsuka, A. (2020) Confidential and Auditable Payments. International Conference on Financial Cryptography and Data Security, Kota Kinabalu, 10-14 February 2020, 466-480. https://doi.org/10.1007/978-3-030-54455-3 33

[11] Androulaki, E., Camenisch, J., Caro, A.D., Dubovitskaya, M., Elkhiyaoui, K. and Tackmann, B. (2020) Privacy-Preserving Auditable Token Payments in a Permissioned Blockchain System. Proceedings of the 2 nd ACM Conference on Advances in Financial Technologies, New York, 21-23 October 2020, 255-267.

https://doi.org/10.1145/3419614.3423259

[12] Cecchetti, E., Zhang, F., Ji, Y., Kosba, A., Juels, A. and Shi, E. (2017) Solidus: Confidential Distributed Ledger Transactions via PVORM. Proceedings of the 2017 ACM SIGSAC Conference on Computer and Communications Security, Dallas, 30 October-3 November 2017, 701-717. https://doi.org/10.1145/3133956.3134010

[13] Chen, Y., Ma, X.C., Tang, C. and Au, M.H. (2020) PGC: Decentralized Confidential Payment System with Auditability. European Symposium on Research in Computer Security, Guildford, 14-18 September 2020, 591-610.

https://doi.org/10.1007/978-3-030-58951-6 29

[14] Naganuma, K., Yoshino, M., Sato, H. and Suzuki, T. (2017) Auditable Zerocoin. 2017 IEEE European Symposium on Security and Privacy Workshops, Paris, 26-28 April 2017, 59-63. https://doi.org/10.1109/EuroSPW.2017.51

[15] Garman, C., Green, M. and Miers, I. (2016) Accountable Privacy for Decentralized Anonymous Payments. International Conference on Financial Cryptography and Data Security, Christ Church, 22-26 February 2016, 81-98. https://doi.org/10.1007/978-3-662-54970-4 5

[16] Narula, N., Vasquez, W. and Virza, M. (2018) zkledger: Privacy-Preserving Auditing for Distributed Ledgers. 15th USENIX Symposium on Networked Systems Design and Implementation, Renton, 9-11 April 2018, 65-80.

[17] Dinh, T.T.A., Saxena, P., Chang, E.-C., Ooi, B.C. and Zhang, C.W. (2015) M2r: Enabling Stronger Privacy in Mapreduce Computation. 24th USENIX Security Symposium (USENIX Security 15), Washington DC, 12-14 August 2015, 447-462.

[18] Zheng, W.T., Dave, A., Beekman, J.G., Popa, R.A., Gonzalez, J.E. and Stoica, I. (2017) Opaque: An Oblivious and Encrypted Distributed Analytics Platform. 14th USENIX Symposium on Networked Systems Design and Implementation, Boston, 27-29 March 2017, 283-298. 
[19] Arasu, A., Blanas, S., Eguro, K., Kaushik, R., Kossmann, D., Ramamurthy, R. and Venkatesan, R. (2013) Orthogonal Security with Cipherbase. CIDR.

[20] Baumann, A., Peinado, M. and Hunt, G. (2015) Shielding Applications from an Untrusted Cloud with Haven. ACM Transactions on Computer Systems (TOCS), 33, 1-26. https://doi.org/10.1145/2799647

[21] Bajaj, S. and Sion, R. (2013) TrustedDB: A Trusted Hardware-Based Database with Privacy and Data Confidentiality. IEEE Transactions on Knowledge and Data Engineering, 26, 752-765. https://doi.org/10.1109/TKDE.2013.38

[22] Schuster, F., Costa, M., Fournet, C., Gkantsidis, C., Peinado, M., Mainar-Ruiz, G. and Russinovich, M. (2015) VC3: Trustworthy Data Analytics in the Cloud Using SGX. 2015 IEEE Symposium on Security and Privacy, San Jose, 21-22 May 2015, 38-54. https://doi.org/10.1109/SP.2015.10

[23] Milutinovic, M., He, W., Wu, H. and Kanwal, M. (2016) Proof of Luck: An Efficient Blockchain Consensus Protocol. Proceedings of the 1st Workshop on System Software for Trusted Execution, Trento, 12-16 December 2016, 1-6. https://doi.org/10.1145/3007788.3007790

[24] Lind, J., Eyal, I., Kelbert, F., Naor, O., Pietzuch, P. and Sirer, E. (2017) Teechain: Scalable Blockchain Payments Using Trusted Execution Environments.

[25] Nakamoto, S. (2019) Bitcoin: A Peer-to-Peer Electronic Cash System. Manubot.

[26] Fedorov, S., Li, W.T. and Karame, G. (2019) Efficient Validation of Transaction Policy Compliance in a Distributed Ledger System. Google Patents, US Patent App. $15 / 916,293$.

[27] Zhao, C.C., Saifuding, D., Tian, H.L., Zhang, Y. and Xing, C.X. (2016) On the Performance of Intel Sgx. 2016 13th Web Information Systems and Applications Conference (WISA), Wuhan, 23-25 September 2016, 184-187. https://doi.org/10.1109/WISA.2016.45

[28] Göttel, C., Pires, R., Rocha, I., Vaucher, S., Felber, P., Pasin, M. and Schiavoni, V. (2018) Security, Performance and Energy Trade-Offs of Hardware-Assisted Memory Protection Mechanisms. 2018 IEEE 37th Symposium on Reliable Distributed Systems (SRDS), Salvador, 2-5 October 2018, 113-142. https://doi.org/10.1109/SRDS.2018.00024

[29] Danezis, G. and Meiklejohn, S. (2015) Centrally Banked Cryptocurrencies. https://doi.org/10.14722/ndss.2016.23187

[30] Walker, P. and Venables, P.J. (2017) Cryptographic Currency for Securities Settlement. Google Patents, US Patent 9,704,143.

[31] Beimel, A. (2011) Secret-Sharing Schemes: A Survey. International Conference on Coding and Cryptology, Qingdao, 30 May-3 June 2011, 11-46.

https://doi.org/10.1007/978-3-642-20901-7 2

[32] Shamir, A. (1979) How to Share a Secret. Communications of the ACM, 22, 612-613. https://doi.org/10.1145/359168.359176

[33] Blakley, G.R. (1979) Safeguarding Cryptographic Keys. International Workshop on Managing Requirements Knowledge (MARK), New York, 4-7 June 1979, 313. https://doi.org/10.1109/MARK.1979.8817296

[34] Shen, Y.R., Tian, H.L., Chen, Y., Chen, K., Wang, R.J., Xu, Y., Xia, Y.B. and Yan, S.M. (2020) Occlum: Secure and Efficient Multitasking inside a Single Enclave of Intel Sgx. Proceedings of the Twenty-Fifth International Conference on Architectural Support for Programming Languages and Operating Systems, Lausanne, 16-20 March 2020, 955-970. https://doi.org/10.1145/3373376.3378469 